\title{
Indicadores de sustentabilidade em medicina laboratorial
}

\section{Sustainability indicators in laboratory medicine}

Claudia Diório Uliani'; Evelyn Rodrigues²; Valéria Aparecida Faria3; Maria Leide Sena Badaró4; Paschoalina Romano ${ }^{5}$; Maria Elizabete Mendes ${ }^{6}$; Nairo Massakazu Sumita ${ }^{7}$

$\quad$ unitermos
Sustentabilidade
Sustentabilidade
empresarial
Indicadores
Ferramentas da qualidade
Benchmarking

\section{resumo}

O artigo apresenta os princípios conceituais sobre desenvolvimento sustentável, sustentabilidade e avalia a evolução e os impactos na economia, no meio ambiente e na sociedade. Discutem-se a aplicabilidade dos conceitos de sustentabilidade empresarial na medicina laboratorial e os desafios inerentes à implantação no laboratório clínico. O impacto dos indicadores de sustentabilidade e seu papel no processo de gestão também são analisados criticamente sob a ótica do balanço socioambiental. O texto apresenta ainda algumas ferramentas para avaliação e interpretação dos indicadores e sua aplicação no processo de análise crítica. Finalmente, o artigo descreve a importância dos indicadores de sustentabilidade na prática do benchmarking e sua aplicabilidade no laboratório clínico.

\section{abstract}

The article presents the conceptual principles on sustainable development and sustainability. Furthermore, it evaluates the progress and impacts on the economy, environment and society. It discusses the applicability of the concepts of corporate sustainability in laboratory medicine and the challenges of deployment in the clinical laboratory. The impact of sustainability indicators and their role in management are also critically reviewed from the perspective of social and environmental balance. Additionally, the text provides some tools for evaluation and interpretation of indicators and their corresponding application in the critical analysis process. Lastly, the article describes the importance of sustainability indicators in the practice of benchmarking and its applicability in the clinical laboratory. key words

Sustainability

Corporate sustainability

Indicators

Quality tools

Benchmarking

1. Administradora de Empresas; gestora de Registro de Não Conformidade, Ação Preventiva e Corretiva da Divisão de Laboratório Central do Hospital das Clínicas da Faculdade de Medicina da Universidade de São Paulo (DLC/HC-FMUSP).

2. Bióloga; gestora de Meio Ambiente da DLC/HC-FMUSP.

3. Bióloga; gestora de Saúde e Segurança Ocupacional da DLC/HC-FMUSP.

4. Bióloga; gestora do Plano de Atendimento à Emergência da DLC/HC-FMUSP.

5. Mestra em Ciências; biologista encarregada da Divisão do Serviço de Bioquímica Clínica do DLC/HC-FMUSP

6. Doutora em Medicina (Patologia); médica patologista clínica; chefe da Seção Técnica de Bioquímica de Sangue da DLC/HC-FMUSP (LIM-03 da Patologia Clínica); coordenadora do Núcelo de Qualidade e Sustentabilidade da DLC/HC.FMUSP.

7. Doutor em Medicina; professor da disciplina de Patologia Clínica da FMUSP; médico patologista clínico; diretor do Serviço de Bioquímica Clínica da DLC/HC-FMUSP (LIM-03 da Patologia Clínica); assessor médico em Bioquímica Clínica do Fleury Medicina e Saúde. 


\section{Introdução}

A discussão acerca da sustentabilidade surgiu há aproximadamente 40 anos e tem como objetivo preconizar ações para suprir as necessidades dos seres humanos sem comprometer o futuro das próximas gerações.

O conceito de desenvolvimento sustentável teve origem nos debates de reavaliação sobre as noções de desenvolvimento ligadas à ideia de crescimento e dos seus limites. Na década de 1970 foi publicado o relatório denominado the limits to growth ${ }^{(6)}$, pelo Clube de Roma, o qual rompia com a ideia de ausência de limites para a exploração dos recursos naturais, em contraponto à teoria desenvolvimentista industrial.

No ano de 1972 foi realizada a Conferência de Estocolmo, na qual se ressaltava que a maioria dos problemas ligados ao meio ambiente ocorria em escala global e se agravava de modo exponencial. As principais preocupações eram o crescimento populacional, a urbanização e a tecnologia envolvida com a industrialização(8).

Com o surgimento do conceito de ecodesenvolvimento ${ }^{(28)}$, passaram a ser estudadas questões como a educação, a participação e a preservação de recursos naturais em conjunto com a satisfação das necessidades básicas, observando-se, em decorrência, a interdependência em nível global entre o desenvolvimento e o meio ambiente.

Em 1987 emergiu o conceito de desenvolvimento sustentável no relatório Our common future ${ }^{(27)}$ da Comissão Mundial de Meio Ambiente e Desenvolvimento, liderada pela norueguesa Gro Harlem Brundtland, onde são detalhados os desafios e esforços comuns para a paz, a segurança, o desenvolvimento e o meio ambiente, com propostas de mudanças legais e institucionais.

Em 1992, houve a conferência do Rio-92, de onde resultaram dois documentos:

- Carta da Terra ou Declaração do Rio, que estabelecia acordos internacionais visando o respeito e a integridade do sistema global de ecologia e desenvolvimento ${ }^{(11)}$;

- Agenda 21, na qual 121 países assumiram o desafio de incorporar em suas políticas públicas os princípios do desenvolvimento sustentável(1).

Em 2002, no encontro Rio+10, foi elaborado o Protocolo de Kioto, o qual firmava o compromisso de países mais industrializados, grandes geradores de resíduos poluentes, serem tributados e responsabilizados pela não preservação do meio ambiente para as gerações futuras. Nele, a cúpula mundial compreendeu e sintetizou o desenvolvimento sustentável com base em três pilares: o econômico, o social e o ambiental, com o objetivo fundamental de erradicar a

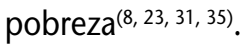

Atualmente, existe o consenso de que há uma nova maneira de a sociedade relacionar-se com o ecossistema, garantindo sua continuidade. Para tanto é necessário reduzir a poluição ambiental, eliminar desperdícios e diminuir a pobreza $^{(6)}$.

\section{Sustentabilidade empresarial}

No âmbito dos negócios, entende-se a sustentabilidade empresarial(26) como a atividade capaz de se manter, por período indeterminado de tempo, apesar dos imprevistos que possam ocorrer, não colocando em riscos os recursos naturais dos quais depende. Assim, a empresa continua a visar lucro, mas passa a considerar o impacto de suas atividades no meio ambiente, procurando amenizá-lo de maneira eficiente, desempenhando, ao mesmo tempo, ações de cunho social, seja em benefício de seus funcionários ou da comunidade ${ }^{(5,14)}$.

No nível empresarial, há uma busca pelo resultado financeiro, pela ampliação de market share e pela sobrevivência e manutenção da competitividade, que se traduz no acirramento da competição em nível mundial, elevação da escala de produção e redução dos custos.

$\mathrm{Na}$ tentativa de reverter esse panorama, as empresas passaram a se reestruturar para reduzir o impacto ambiental e a ampliar as ações de responsabilidade social. Há consenso de que a sustentabilidade empresarial é um processo evolutivo e resulta da combinação de três vertentes de desenvolvimento institucional, isto é, pessoas, planeta e lucro. As ações têm por objetivo beneficiar não somente a geração presente, mas principalmente a futura, agregada ao crescimento da economia e à melhoria da qualidade, do ambiente e da sociedade ${ }^{(35)}$.

Segundo a Comissão Mundial do Meio Ambiente ${ }^{(27)}$, o desafio do desenvolvimento sustentável é trazer as considerações ambientais para o centro das tomadas de decisões econômicas, planejando o futuro em todos os níveis.

A velocidade de implantação de um modelo de desenvolvimento sustentável depende da vontade coletiva dos cidadãos de cada região para vencer a inércia das estruturas e processos preexistentes. Assim, a vantagem competitiva visa associar a imagem da organização à consciência ecológica ${ }^{(23)}$. Os produtos ecologicamente corretos agregam em seus preços mais recursos tecnológicos, menos desperdícios e menor nível de geração de poluição. 
Para Tachizawa ${ }^{(32)}$, um dos maiores desafios que o mundo enfrentará nesse novo milênio será fazer que as forças de mercado protejam e melhorem a qualidade do ambiente. Para isso é fundamental a definição de padrões baseados no desempenho e no uso criterioso de instrumentos econômicos, em um contexto harmonioso da regulamentação. Esse novo cenário econômico se caracterizará por uma rígida postura dos clientes, voltada à expectativa de interagir com organizações que sejam éticas, detentoras de uma boa imagem institucional e que atuem de forma ecologicamente responsável(2).

Os impactos das atividades em um laboratório clínico afetam os acionistas, os funcionários, os fornecedores, os consumidores e também a comunidade da região em que se localiza, os quais, no conjunto, constituem uma rede de stakeholders. Há uma conjugação de fatores indicando que os laboratórios não podem mais se preocupar, unicamente, com a obtenção do lucro, pois as suas atividades não se restringem apenas ao âmbito econômico, elas também geram efeitos no meio ambiente e na sociedade ${ }^{(19)}$.

À medida que este conceito se tornou mais difundido $\mathrm{e}$ aplicado na prática operacional das empresas, entre elas os laboratórios clínicos, passaram-se a estabelecer objetivos, metas e os indicadores para monitorar o planejamento da sustentabilidade institucional. Assim, tornou-se imprescindível que os laboratórios clínicos também passassem a se preocupar como as demais instituições em evitar impactos ambientais.

Segundo Mendes (21), a aplicação desse conceito no laboratório resulta no desenvolvimento econômico e material, sem agredir o meio ambiente, em razão da utilização dos recursos naturais de forma inteligente, para que estes sejam preservados para o futuro.

A atividade laboratorial, cada vez mais focada na rentabilidade associada à responsabilidade socioambiental, faz uso da sustentabilidade como importante componente estratégico. Para isso, aplica os princípios já consolidados como ecologicamente corretos, economicamente viáveis, socialmente justos e culturalmente aceitos.

\section{Indicadores}

No contexto do desenvolvimento sustentável, os indicadores ambientais passaram a ter importância significativa por fornecerem evidências objetivas para as intervenções, as quais se tornaram imediatas quando envolviam questões relacionadas com os recursos naturais. Os indicadores servem de suporte para a tomada de decisões, auxiliando os gestores na atribuição de fundos, alocação de recursos naturais e definição de prioridades. As principais funções dos indicadores são avaliar condições e tendências, permitir a comparação entre processos e situações, revelar tendências em relação às metas e aos objetivos, provendo informações de advertência, e antecipar futuras condições ${ }^{(21,22,29,31)}$.

Relativamente a conteúdo, amplitude e natureza do sistema de indicadores sustentáveis, consideram-se quatro categorias: ambientais, econômicas, sociais e institucionais.

O conjunto de indicadores, segundo Elkington ${ }^{(13)}$, propicia a comparação de condições em diferentes locais ou áreas geográficas: a informação sobre o nível de cumprimento das normas ou critérios legais, a análise de dados para detectar tendências no tempo e no espaço, as aplicações em desenvolvimentos científicos, servindo de alerta para a necessidade de investigação mais aprofundada e a informação ao público sobre os processos de desenvolvimento sustentável(8, 31,35). A publicação da Organization for Economic Corporation and Development (OECD) ${ }^{(24)}$ forneceu o primeiro mecanismo para monitoramento do progresso ambiental para os países participantes da instituição, com o objetivo de monitorar o ambiente e as mudanças climáticas, integrando as preocupações ambientais com as políticas públicas e econômicas.

A NBR ISO 14031:2004(4) aborda os Indicadores de Desempenho Gerenciais (IDG), os quais geram informações relativas à gestão da empresa, que influenciam positivamente o seu desempenho ambiental. Ela aponta os Indicadores de Desempenho Operacional (IDO), que proporcionam informações relacionadas com as operações do processo produtivo do laboratório, com reflexos no seu desempenho ambiental. Os indicadores refletem os impactos econômico, ecológico e social ${ }^{(2)}$.

A United Nations Statistics Division (UNSD), uma divisão de estatística da Organização das Nações Unidas (ONU), visando abordar a questão ambiental nos sistemas de mensuração econômica, desenvolveu um sistema para integrar os vários sistemas atualmente utilizados. De acordo com alguns estudos, um sistema de contas verdes não só é realizável, como também pode fornecer informações importantes em termos de desenvolvimento de políticas e planejamento $^{(21,22,29,31)}$.

A elaboração de relatórios de sustentabilidade tem sido praticada por alguns laboratórios clínicos no Brasil, os quais divulgam o balanço de suas ações no campo socioambiental. Existem modelos que auxiliam na elaboração desses relatórios. A Global Reporting Initiative (GRI) é um 
exemplo no âmbito internacional. No Brasil, os exemplos são o Instituto Ethos e o Instituto Brasileiro de Análises Sociais e Econômicas (IBASE).

O Sustainability Reporting Guidelines (GRI) ${ }^{(31)}$, reconhecido e adotado em todo mundo, considera que os indicadores de sustentabilidade fornecem informações importantes sobre a situação econômica e o desempenho ambiental e social da organização. Segundo o documento, o relatório de sustentabilidade, apoiado nesses indicadores, é útil na prática de benchmarking, particularmente no que diz respeito a normas, leis, padrões de desempenho e iniciativas voluntárias. Além disso, demonstra como a empresa é capaz de influenciar e ser influenciada pelas expectativas em relação à sustentabilidade, quando se fazem comparações dentro da mesma empresa e entre diferentes organizações.

\section{Indicadores de sustentabilidade no laboratório}

Os indicadores de sustentabilidade auxiliam os tomadores de decisão a avaliar os resultados práticos, resultantes das ações voltadas para este assunto no laboratório clínico. Com base nos resultados apontados por eles, os gestores laboratoriais planejam estratégias que favoreceçam a meIhoria do sistema.

Os sistemas de indicadores podem utilizar algumas destas ferramentas para avaliar os esforços do laboratório no sentido da implantação do desenvolvimento sustentável:

- ecological footprint method (pegada ecológica) representa o espaço ecológico correspondente que sustenta determinado sistema. É uma técnica analítica e educacional, pois contribui para a conscientização da sociedade sobre os problemas ambientais;

- dashboard of sustainability (painel de controle) - esse indicador é constituído de medidas agregadas nas três dimensões da sustentabilidade: a econômica, a social e a ambiental. A representação gráfica do indicador é semelhante à de um painel de controle de um carro, com três mostradores, um para cada dimensão;

- barometer of sustainability (barômetro de sustentabilidade) - permite a combinação de indicadores e alcança os resultados por meio de índices. Sua principal característica é a capacidade de agregar indicadores, utilizando muitos dados, por vezes contraditórios. Uma escala de desempenho avalia a importância de cada índice para o indicador com base nos outros índices existentes.
A apreciação do desenvolvimento sustentável desde a escolha dos indicadores até a comunicação de resultados foi sintetizada nos 10 princípios de Bellagio ${ }^{(8)}$.

A avaliação de um sistema, rumo à sustentabilidade, deve ter uma visão clara do que se pretende alcançar e quais são as metas definidas. Deve incorporar uma perspectiva holística, restringindo-se o tempo de avaliação e o escopo das medições ${ }^{(25)}$.

A análise das ações rumo à sustentabilidade deve ter foco prático $^{(7)}$. A construção dos dados ${ }^{(19,33)}$ é feita de modo a torná-los acessíveis e explícitos, atendendo às necessidades dos usuários. Deve-se procurar uma forma simples de comunicação, para que esta seja efetiva junto aos envolvidos.

É preciso garantir a participação dos tomadores de decisão para assegurar uma forte adoção de políticas e nos resultados da ação.

O desenvolvimento da capacidade de determinar tendências a partir de medidas repetidas dos indicadores ou pela adaptação e ajuste das metas auxilia na promoção de um aprendizado coletivo. A capacidade da instituição em coletar, manter e documentar as informações e dados é condição essencial para se atingir os objetivos.

O conjunto de indicadores para análise comparativa na medicina laboratorial atende às três dimensões: ambiental, social e econômica.

\section{Indicadores da dimensão ambiental}

- Consumo de papéis (número de caixas consumidas) por mês/número de exames realizados por mês.

- Consumo de energia elétrica (kVA) por mês/número de exames realizados por mês.

- Consumo de água $\left(\mathrm{m}^{3}\right)$ por mês/número de exames realizados por mês.

- Consumo de gás $\left(\mathrm{m}^{3}\right)$ por mês/número de exames realizados por mês.

- Total de resíduos gerados $(\mathrm{kg})$ por mês/número de exames realizados por mês.

- Quantidade de material reciclado por mês/número de exames realizados por mês.

- Número de lâmpadas de mercúrio trocadas e descartadas/mês.

\section{Indicadores da dimensão social}

- Indicadores funcionais:

$\sqrt{ }$ número de empregos diretos gerados;

$\sqrt{ }$ número de admissões/ano; 
$\sqrt{ }$ número de demissões/ano;

$\checkmark$ produtividade total;

$\sqrt{ }$ absenteísmo;

$\sqrt{ }$ número de horas extras/mês.

- Indicadores sociais internos:

$\sqrt{ }$ frequência de exames periódicos;

$\sqrt{ }$ carga de treinamentos realizados/mês.

- Indicadores de segurança no trabalho:

$\sqrt{ }$ número de acidentes do trabalho com e sem afastamentos por mês;

$\sqrt{ }$ taxa de frequência $=\mathrm{n}^{\circ}$ de comunicado de acidente do trabalho (CAT) $\times 10^{6} / \mathrm{n}^{\circ}$ total de horas homem trabalhado;

$\sqrt{ }$ taxa de gravidade $=\mathrm{n}^{\circ}$ de dias de afastamento $\times 10^{6} / n^{\circ}$ total de horas homem trabalhado;

$\sqrt{ }$ número de brigadistas formados;

$\sqrt{ }$ CAT: número de comunicado de acidente do trabalho/mês.

- Indicadores sociais na comunidade:

$\sqrt{ }$ número de ações culturais promovidas por ano;

$\sqrt{ }$ número de parcerias efetuadas com organizações não governamentais por ano;

$\sqrt{ }$ número de ações filantrópicas efetuadas por ano;

$\sqrt{ }$ número de eventos socioambientais patrocinados por ano.

\section{Indicadores da dimensão econômica}

- Produção:

$\sqrt{ }$ número de exames realizados por ano.

- Faturamento:

$\sqrt{ }$ valor total das vendas por mês.

- Investimentos:

$\sqrt{ }$ aplicações em dinheiro realizadas por mês.

- Receita:

$\sqrt{ }$ quantia recebida por mês.

- Tributos:

$\sqrt{ }$ impostos e taxas pagos ao governo por mês.
É recomendável que a direção do laboratório clínico faça a análise do sistema de gestão em intervalos planejados para garantir a adequação contínua, a pertinência e a eficácia ${ }^{(3)}$. Nela se avaliam os resultados do desempenho, suas oportunidades de melhoria e eventuais necessidades de alterações.

\section{Benchmarking}

É um processo sistemático para identificar, compreender e avaliar processos de trabalho das organizações, que são reconhecidas como representantes das melhores práticas, com o propósito de realizar, aperfeiçoar procedimentos e introduzir novos métodos. A motivação para a aplicação dessa técnica está em melhorar e reduzir lacunas em relação ao referencial escolhido ${ }^{(34)}$. A inclusão dessa prática traz incrementos consideráveis nas ações de melhoria quando em comparação com organizações que não a utilizam. O benchmarking age como uma ferramenta para propor metas, utilizando-se de normas externas e objetivas para inovar a gestão por meio da identificação de problemas específicos e de novas oportunidades.

A técnica de referenciação consiste em uma abordagem estruturada e contínua que envolve medição, avaliação e comparação de resultados. Na etapa das medições é importante que haja a padronização na obtenção dos dados dos indicadores para que os resultados sejam comparáveis de forma homogênea(12, 15-17).

As etapas de sua aplicação compreendem: identificação do propósito da sua realização, criação da equipe, identificação das instituições que serão referência, definição da metodologia de trabalho, coleta de dados, comparação e estabelecimento de diferenças, plano de ação para implantar novo processo ${ }^{(18)}$.

Lavorato $^{(18)}$ discute o benchmarking ambiental como uma forma de aprendizado mediante as comparações competitivas, com ênfase nos resultados das empresas de referência ambiental. Advindo dessa prática, verificam-se melhorias na conscientização ambiental dos funcionários, diminuição da poluição, redução de custos devida à minimização do desperdício e otimização da percepção externa da imagem institucional.

Em 2003 foi realizado o primeiro benchmarking ambiental brasileiro com o objetivo de reunir, selecionar e premiar as melhores práticas ambientais ${ }^{(18)}$. A divulgação dos resultados dos dados relativos às ações de sustentabilidade pela empresa possibilita a aplicação correta da técnica de referenciação ${ }^{(5)}$. A análise decorrente dessa prática traduz o interesse da instituição em melhorar as práticas de sustentabilidade. 
O estabelecimento de cri térios objetivos para a seleção das melhores práticas ambientais permite que as demais empresas aprendam e moldem as soluções encontradas para os impactos ambientais provocados pelo seu processo produtivo. Além disso, novas ideias podem ser desenvolvidas, representando um alcance mais rápido e efetivo da qualidade ambiental de produtos, processos e serviços.

O cumprimento da legislação, como o licenciamento ambiental da empresa, é um dos requisitos das boas práticas ambientais.

Outra iniciativa é o Programa de Atuação Responsável, que corresponde à versão brasileira do Responsible Care Program, criado no Canadá, pela Canadian Chemical Producers Association, implantado em mais de 40 países com indústrias químicas a partir de 1985 . Este programa foi estruturado com base na melhoria contínua e envolve a segurança de processos, a saúde e a segurança do trabalhador, a proteção ambiental, o transporte e a distribuição, o gerenciamento do produto, o diálogo com a comunidade e a preparação e o atendimento de emergências. Ele estabelece mecanismos que permitam o desenvolvimento de sistemas e metodologias adequados para a gestão ambiental do setor químico/petroquímico e conta com o apoio da Associação Brasileira da Indústria Química (ABIQUIM).

A implantação dos conceitos descritos na norma internacional SA 8000, que define os requisitos relativos às práticas sociais do emprego por fabricantes e seus fornecedores, é considerada mundialmente a norma mais aplicável ao ambiente de trabalho. Ela requer a adoção de uma postura ética e transparente das operações da empresa e da sua cadeia de fornecimento.

A prática do marketing verde ou ambiental consiste no desenvolvimento de atividades para gerar e facilitar quaisquer trocas com a intenção de satisfazer os desejos e necessidades dos consumidores, com o mínimo de impacto negativo sobre o meio ambiente. Ele incorpora a preocupação ambiental e contribui para a conscientização ambiental pelos consumidores. Ao adotar o marketing verde, a organização informa seus consumidores sobre as vantagens de se adquirir produtos e serviços ambientalmente responsáveis, de forma a estimular e despertar o desejo do mercado por esta categoria de produtos.

Observam-se, ainda, como exemplos de melhores práticas ambientais: a implantação de sistemas de gestão ambiental baseados na norma ambiental ${ }^{(3)}$ ABNT/NBR ISO 14001 e os estudos de impacto ambiental, com a elaboração do relatório de impacto ambiental.

A divulgação, pela empresa, dos resultados dos dados relativos às ações de sustentabilidade possibilita a aplicação correta da técnica de referenciação ${ }^{(5)}$. A análise decorrente dessa prática traduz o interesse da instituição em melhorar as práticas de sustentabilidade.

\section{Conclusão}

O conceito de desenvolvimento sustentável é relativamente novo, assim como recentes são as tentativas de criarem-se sistemas que capturem a nova maneira de entender o processo.

É necessário aprofundar os conhecimentos nesse campo para que haja transformação dessas ferramentas em suporte à decisão, nas esferas social, pública e privada, orientando o desenvolvimento para uma direção mais sustentável.

A implantação dos conceitos de sustentabilidade empresarial no laboratório clínico é factível, e a decisão pela implantação é de responsabilidade da alta direção. $\mathrm{O}$ projeto necessita estar ancorado em um planejamento meticuloso, sendo fundamentais a disciplina e o esforço de todos os envolvidos para se alcançarem os objetivos.

O conjunto de indicadores sugerido neste artigo é abrangente e utiliza informações acessíveis, de fácil obtenção, sem a necessidade de ferramentas dispendiosas para a operacionalização, fato que torna possível sua aplicação em laboratórios clínicos de tamanhos distintos e das diferentes esferas: público, privado, hospitalar, ambulatorial.

A interpretação e a análise dos indicadores proporcionarão aos gestores os instrumentos adequados para a tomada de decisões rumo ao laboratório sustentável e uma consciência ambiental ampliada, cumprindo assim a função social da patologia clínica.

\section{Referências}

1. AGENDA 21. Disponível em: <http://www.agenda21local. com.br/abe1.htm.> Acesso em: 12 maio 2011.

2. ARAÚJO, G. C. et al. Sustentabilidade empresarial: conceito e indicadores. 2006. Disponível em: <http://www. convibra.com.br/2006/artigos/61_pdf.pdf>. Acesso em: 28 abr. 2011.

3. ASSOCIAÇÃO BRASILEIRA DE NORMAS TÉCNICAS (ABNT). NBR ISO 14001:2004, 2. ed. 31/12/2004, válida a 
partir de 31/01/2005 - Sistema da gestão ambiental requisitos com orientações para uso, 27 páginas.

4. ASSOCIAÇÃO BRASILEIRA DE NORMAS TÉCNICAS (ABNT). NBR ISO 14031:2004, 1. ed. 27/02/2004, válida a partir de 29/03/2004 - Gestão ambiental - Avaliação de desempenho ambiental - Diretrizes, 38 páginas.

5. AZEVEDO, A. L. V. Indicadores de sustentabilidade empresarial no Brasil: uma avaliação do relatório do CEBDS. Revista Iberoamericana de Economía Ecológica, v. 5, p. 75-93, 2011. Disponível em: <http://www.redibec. org/IVO/rev5_06.pdf.> Acesso em: 12 mai 2011.

6. BARONI, M. Ambigüidades e deficiências do conceito de sustentabilidade. RAE, v. 32, n. 2, p. 14-24, abr-jun 1992.

7. BELL, S.; MORSE, S. Sustainability indicators: measuring the immeasurable. London, UK: Earthscan, 1999.

8. BELLEN, H. M. Indicadores de sustentabilidade. Rio de Janeiro: FGV, 2006.

9. BERLITZ, F. A.; HAUSSEN, M. L. Indicadores de desempenho da fase analítica. In: OLIVEIRA, C. A.; MENDES, M. E. Gestão da fase analítica do laboratório. 1. ed. Rio de Janeiro: Control Lab, 2010. cap.5. p. 119-42.

10. CAMPBELL, A. B. Benchmarking: a performance intervention tool. In: Joint Commission on Accreditation of Healthcare organizations. 2. ed. Ilinois: Joint Commission Resources, v. 20, n. 5, p. 225-8, 1994.

11. CARTA DA TERRA. Disponível em: $<$ http://www.cartadaterrabrasil. org/prt/text.html>. Acesso em: 10 maio 2011.

12. CAVALCANTI, C.; BEGOSSI, A. Meio ambiente, desenvolvimento sustentável e políticas públicas. São Paulo: Cortez Editora, 1997.

13. ELKINGTON, J. Cannibals with Forks: the triple bottom line of $21^{\text {st }}$ century business. Universidade de Michigan: New Society Publishers, 1998.

14. FARRELL, A; HART, M. What does sustainability really mean? Environment, v. 44, n. 9, p. 4-9, 1998.

15. GALORO, C. A. O. A aplicação da técnica de referenciação (benchmarking) em serviços de medicina laboratorial. São Paulo, 2008. Tese (doutoramento) - Departamento de Patologia, Faculdade de Medicina da Universidade de São Paulo.

16. GIFT, R.; MOSEL, D. Benchmarking in health care: a collaborrative approach. American Hospital Publishing Inc., 1994.

17. GUIMARÃES, R. P. Desenvolvimento sustentável: da retórica à formulação de políticas: In: BECKER, B. K.; MIRANDA, M. A geografia política do desenvolvimento sustentável. Rio de Janeiro: UFRJ, 1997.

18. LAVORATO, M. L. A. As vantagens do benchmarking ambiental. Revista Cientifica Eletrônica da Engenharia de Produção, v. 4, n. 2, 2003. Disponível em: $<$ http://producaoonline.org.br/index.php/rpo/article/ viewArticle/307>. Acesso em: 10 maio 2011.

19. MEADOWS, D. Indicators and informations systems for sustainable development. Hartland Four Corners: The Sustainability Institute, 1998.

20. MENDES, M. E. et al. Gestão por processos no laboratório clínico: uma abordagem prática. São Paulo: EPR Editora Ltda., 2007.

21. MENDES, M. E. Sustentabilidade na medicina laboratorial. 188. ed. Laes\&Haes, 2011.

22. NEVALAINEN, D. et al. Evaluating laboratory performance on quality indicators with the six sigma scale. Arch Pathol Lab Med, v. 124, n. 4, p.519-9, 2000.
23. OLIVEIRA-FILHO J. E. Gestão ambiental e sustentabilidade: um novo paradigma eco-econômico para as organizações modernas. Rev Teor Pol Soc Cidad, v. 1, n. 1, p. 1-21. Disponível em: <http://www.fbb.br/downloads/domus_ jaime.pdf>. Acesso em: 13 maio 2011.

24. ORGANIZATION FOR ECONOMIC CORPORATION AND DEVELOPMENT (OECD). Organization for Economic Corporation and Development: core set of indicators for environmental performance reviews; a synthesis report by the group on the state of the environment. Paris: OECD, 1993.

25. PARRIS, T. M.; KATES, R. W. Characterizing and measuring sustainable development. Annu Rev Environ Resour, v. 28, p. 13.1-13.28, 2003.

26. PHILIPPI, L. S. A construção do desenvolvimento sustentável. In: LEITE, A. L. T. A. Questões ambientais: conceitos, história, problemas e alternativa. 2. ed. v. 5. Brasília: Ministério do Meio Ambiente, 2001.

27. REPORT of the World Commission on Environment and Development: our Common Future. UN Documents: Gathering a Body of Global Agreements. United Nations Documents, document A/42/A427, 1987. Disponível em: <http://www.un-documents.net/wced-ocf.htm>. Acesso em: 18 abr. 2011.

28. SACHS, I. Estratégias de transição para o século XXI: desenvolvimento e meio ambiente. São Paulo: Studio Nobel, Fundação do Desenvolvimento Administrativo, 1993.

29. SAVITZ, A. W.; WEBER, K. The triple bottom line: how today's best-run companies are achieving economic, social and environmental success - and how you can too. San Francisco: Jossey-Bass, 2006.

30. SIMFRONIO, T. Responsabilidade social corporativa: um estudo de aplicação e resultados da Norma SA8000 no Brasil. Disponível em: <http://www.inclusion.com.br/ sa8000_aplicacao_e_resultado_no_brasil.pdf $>$. Acesso em: 11 maio 2011.

31. SUSTAINABILITY REPORTING GUIDELINES. Version 3.1, 2000-2011. Disponível em: <http://www.globalreporting. org/NewsEventsPress/PressResources/2011/G3.1Sust ainabilityReportingMostCompleteGuidanceEver.htm>. Acesso em: 18 abr. 2011.

32. TACHIZAWA, T.; DE ANDRADE, R. O. B.; CARVALHO, A. B. Gestão ambiental: enfoque estratégico aplicado ao desenvolvimento sustentável. 2. ed. São Paulo: Pearson Education do Brasil, 2002. 232p.

33. UN Div. Sustain. Dev. Indicators of sustainable development: framework and methodologie. Backgr. Pap. 3, $9^{\text {th }}$ Sess. Comm. Sustain. Dev., New York, Apr. 16-2001.

34. VORHIES, DW.; MORGAN, NA. Benchmarking marketing capabilities for sustainable competitive advantage. The Journal of Marketing, v. 69, n. 1, p. 80-94, 2005.

35. WILLARD, B. The sustainability advantage: seven business case benefits of a triple bottom line. Canada: New Society Publishers, 2002.

Endereço para correspondência

Maria Elizabete Mendes

Divisão de Laboratório Central do HC-FMUSP Núcleo da Qualidade Av. Dr. Enéas de Carvalho Aguiar, 155 - Prédio dos Ambulatórios $-2^{\circ}$ andar - Bloco 9 CEP: 05403-010 - São Paulo-SP e-mail: ccq.dlc@hcnet.usp.br 